Discrete Comput Geom 29:561-573 (2003)

DOI: $10.1007 / \mathrm{s} 00454-002-0790-3$

\title{
The Characteristic Intersection Property of Line-Free Choquet Simplices in $E^{d}$
}

\author{
Valeriu Soltan \\ Department of Mathematical Sciences, George Mason University, \\ 4400 University Drive, Fairfax, VA 22030-4444, USA \\ vsoltan@gmu.edu
}

\begin{abstract}
Generalizing the characteristic intersection property of Choquet simplices, it is proved that for line-free convex bodies $B_{1}$ and $B_{2}$ in $E^{d}$, the following conditions are equivalent: (i) there is a line-free convex body $B \subset E^{d}$ such that every nonempty intersection $B_{1} \cap\left(v+B_{2}\right), v \in E^{d}$, is a homothetic copy of $B$, (ii) both $B_{1}$ and $B_{2}$ are Choquet simplices and the nonempty intersections $B_{1} \cap\left(v+B_{2}\right), v \in E^{d}$, are homothetic copies of a Choquet simplex $B$. All such triplets $B_{1}, B_{2}, B$ are described.
\end{abstract}

\section{Introduction}

In what follows, by a convex body we mean a closed convex set with nonempty interior in the Euclidean space $E^{d}$. We will distinguish bounded and unbounded convex bodies. A set in $E^{d}$ is called line-free provided it contains no line. By a $d$-polyhedron (in particular, a $d$-polytope, a $d$-simplex, or a simplicial $d$-cone) we mean a convex polyhedral set of dimension $d$.

In 1956 Choquet [2] defined a simplex (afterwards called a Choquet simplex) as a convex set $S$ in a linear space $E$ such that for any two homothetic copies of $S$ their intersection, if nonempty, is again a homothetic copy of $S$ (possibly degenerated into a point):

$$
(u+\lambda S) \cap(v+\mu S)=w+v S, \quad u, v, w \in E, \quad \lambda, \mu, v \geq 0 .
$$

From [1] and [6] it follows that a line-free convex body $B \subset E^{d}$ is a Choquet simplex if and only if $B$ is either a usual $d$-simplex of the form $\operatorname{conv}\left(x_{0}, x_{1}, \ldots, x_{d}\right)$ or a simplicial $d$-cone of the form $B=\sum_{i=1}^{d}\left[x_{0}, x_{i}\right)$, where $x_{0}, x_{1}, \ldots, x_{d}$ are affinely independent points in $E^{d}$ and $[x, z)$ means the half-line through $z$ with the endpoint $x$.

As was mentioned in [4], the above definition can be slightly modified in the following way: a line-free convex body $B \subset E^{d}$ is a Choquet simplex if and only if every nonempty 
intersection of $B$ and a translate of $B$ is a homothetic copy of $B$ (possibly degenerated into a point):

$$
B \cap(v+B)=w+\lambda B, \quad v, w \in E^{d}, \quad \lambda \geq 0 .
$$

The following generalization of the characteristic intersection property of Choquet simplices was obtained in [7].

Theorem 1. Let $B_{1}$ and $B_{2}$ be a pair of bounded convex bodies in $E^{d}$ such that the nonempty intersections $B_{1} \cap\left(v+B_{2}\right), v \in E^{d}$, are homothetic copies (possibly degenerated into points) of a given bounded convex body $B \subset E^{d}$. Then $B_{1}, B_{2}$, and $B$ are homothetic Choquet simplices, i.e., they are homothetic $d$-simplices.

Our purpose here is to extend this result for the case of line-free convex bodies in $E^{d}$ (see Theorem 2).

Let us recall that a boundary point $x$ (or a boundary half-line $l$ ) of a convex body $B \subset E^{d}$ is called exposed provided there is a hyperplane $H$ such that $B \cap H=\{x\}$ (respectively, $B \cap H=\{l\}$ ). The set of exposed points of a convex body $B$ is denoted $\exp B$. It is well known (see [5] and [8]) that a bounded convex body in $E^{d}$ is the closed convex hull of its exposed points, and an unbounded line-free convex body in $E^{d}$ is the closed convex hull of the union of its exposed points and exposed half-lines. As a result, any line-free convex body in $E^{d}$ has at least one exposed point.

A boundary point $x$ of a convex body $B \subset E^{d}$ is called regular if there is a unique hyperplane supporting $B$ at $x$. Denote by $N(B)$ the family of outward unit normals to the convex body $B$ at its regular points. In particular, a convex body $B \subset E^{d}$ is a $d$-polyhedron if and only if the set $N(B)$ is finite.

The characteristic cone $C(B)$ of a convex body $B \subset E^{d}$ (also known as the recession cone) is defined by $C(B)=\left\{y \in E^{d}: x+\lambda y \in B\right.$ for every $\lambda \geq 0$ and $\left.x \in B\right\}$. Finally, $U_{r}(a)=\left\{x \in E^{d}:\|x-a\| \leq r\right\}$ stands for the closed ball with radius $r$ and center $a$.

\section{Main Result}

The main result of this paper is given in Theorem 2 below. For a boundary point $x$ of a convex body $B \subset E^{d}$, we define the tangent cone $T_{x}(B)$ as the closure of the union of the half-lines $[x, z)$, where $z \in B \backslash\{x\}$. It is easily seen that $T_{x}(B)$ is a closed convex cone with apex $x$, distinct from $E^{d}$, and $T_{x}(B)=T_{x}\left(B \cap U_{r}(x)\right)$ for any $r>0$.

Theorem 2. For a pair of line-free convex bodies $B_{1}$ and $B_{2}$ in $E^{d}$, conditions (1) and (2) below are equivalent.

(1) There is a line-free convex body $B \subset E^{d}$ such that every nonempty intersection $B_{1} \cap\left(v+B_{2}\right), v \in E^{d}$, is a homothetic copy of $B$ (possibly degenerated into a point).

(2) There is a Choquet simplex $B$, i.e., $B$ is a $d$-simplex of the form $B=\operatorname{conv}\left(x_{0}, x_{1}\right.$, $\left.\ldots, x_{d}\right)$ or a simplicial $d$-cone of the form $B=\sum_{i=1}^{d}\left[x_{0}, x_{i}\right)$, where $x_{0}, x_{1}, \ldots$, 
$x_{d}$ are affinely independent points, such that one of the following conditions holds:

(a) Both $B_{1}$ and $B_{2}$ are homothetic copies of $B$.

(b) One of $B_{1}, B_{2}$, say $B_{1}$, is a homothetic copy of $B$, and $B_{2}$ is a simplicial $d$ cone that is translate of a tangent cone $T_{x}(B)$, where $x \in\left\{x_{0}, x_{1}, \ldots, x_{d}\right\}$.

(c) Both $B_{1}$ and $B_{2}$ are simplicial $d$-cones that are translates of tangent cones $T_{x}(B)$ and $T_{z}(B)$, where $x$ and $z$ are distinct points in $\left\{x_{0}, x_{1}, \ldots, x_{d}\right\}$ (this condition is possible only if $B$ is a d-simplex).

\section{Auxiliary Lemmas}

We say that points $x \in \exp C$ and $z \in \exp D$ of convex bodies $C$ and $D$ in $E^{d}$ correspond to each other provided the tangent cone $T_{x}(C)$ is a translate of the tangent cone $T_{z}(D)$. We will need the following lemma on tangent cones.

Lemma 1. If $a$ and $b$ are distinct exposed points of a convex body $D \subset E^{d}$, then none of the cones $T_{a}(D),(a-b)+T_{b}(D)$ lies in the other.

Proof. Indeed, assume, for contradiction, that $(a-b)+T_{b}(D) \subset T_{a}(D)$ (the case $T_{a}(D) \subset(a-b)+T_{b}(D)$ is similar). Let $H$ be a hyperplane with the property $D \cap H=$ $\{a\}$. Clearly, $H$ supports the cone $T_{a}(D)$. Then the hyperplane $(b-a)+H$ supports the cone $T_{b}(D)$, and, as a result, $(b-a)+H$ supports $D$ at $b$. Two parallel hyperplanes, $H$ and $(b-a)+H$, both supporting $D$ from the same side, should coincide: $H=(b-a)+H$. Finally, $\{a\}=D \cap H=D \cap(b-a+H)=\{b\}$, a contradiction.

Lemma 2. Under condition (1) of Theorem 2, any exposed point of $B_{1}$ (respectively, of $\left.B_{2}\right)$ corresponds to an exposed point of $B$. Moreover, if $B_{1}$ is bounded, then any exposed point of $B$ corresponds to an exposed point of $B_{1}$.

Proof. Let $z \in \exp B_{1}$ (the case $z \in \exp B_{2}$ is similar). Choose a vector $v \in E^{d}$ such that $z \in \operatorname{int}\left(v+B_{2}\right)$ and consider the intersection $C=B_{1} \cap\left(v+B_{2}\right)$. Clearly, $z$ is an exposed point of $C$ and $T_{z}(C)=T_{z}\left(B_{1}\right)$. Since $C=w+\lambda B$ for some $w \in E^{d}$ and $\lambda>0$ (by condition (1)), the exposed point $z$ of $B_{1}$ corresponds to the exposed point $u=\lambda^{-1}(z-w)$ of $B$.

Now assume that $B_{1}$ is bounded, and let $z \in \exp B$. Denote by $H$ a hyperplane in $E^{d}$ with the property $B \cap H=\{z\}$, and let $H_{1}$ be a translate of $H$ that supports $B_{1}$ at some point $u$ such that $B_{1}$ lies on the same side of $H_{1}$ as $B$ with respect to $H$ (this is possible because $B_{1}$ is bounded). Choose a vector $v \in E^{d}$ such that $u \in \operatorname{int}\left(v+B_{2}\right)$ and consider the intersection $C=B_{1} \cap\left(v+B_{2}\right)$. Since $C$ is homothetic to $B$ (by condition (1)) and since $H_{1}$ supports $C$, the intersection $C \cap H_{1}$ consists of a single point. From the inclusion $u \in B_{1} \cap H_{1}$ we get $C \cap H_{1}=\{u\}$. Hence $u \in \exp C$ and $T_{u}(C)$ is a translate of $T_{z}(B)$. Clearly, $T_{u}\left(B_{1}\right)=T_{u}(C)$. Thus $z$ corresponds to the exposed point $u$ of $B_{1}$. 
Lemma 3. Under condition (1) of Theorem 2, if $B$ is bounded and $B_{2}$ is unbounded, then for any vector $v \in E^{d}$ the set int $B_{1}$ contains at most one exposed point of the body $v+B_{2}$.

Proof. Assume, for contradiction, the existence of a vector $v \in E^{d}$ and distinct exposed points $p, q$ of $B_{2}$ such that int $B_{1}$ contains both $p^{\prime}=v+p$ and $q^{\prime}=v+q$, which are exposed points of $v+B_{2}$. Denote by $H_{p}$ and $H_{q}$ some closed half-spaces in $E^{d}$ with the property $B_{2} \cap H_{p}=\{p\}$ and $B_{2} \cap H_{q}=\{q\}$. Clearly,

$$
\left(v+B_{2}\right) \cap\left(v+H_{p}\right)=\left\{p^{\prime}\right\} \text { and }\left(v+B_{2}\right) \cap\left(v+H_{q}\right)=\left\{q^{\prime}\right\} .
$$

Since $B_{2}$ is unbounded, there is a half-line $l \subset B_{2}$ with the endpoint $\frac{1}{2}(p+q)$. Let $a$ be the unit vector in the direction of $l$.

Put $C=B_{1} \cap\left(v+B_{2}\right)$ and denote by $m$ the intersection of $C$ with the half-line $v+l$. Since $C$ is homothetic to $B$, which is bounded, $m$ is a line segment.

Now choose a real number $\varepsilon>0$ so small that int $B_{1}$ still contains both exposed points $p^{\prime \prime}=v+\varepsilon a+p$ and $q^{\prime \prime}=v+\varepsilon a+q$ of the body $v+\varepsilon a+B_{2}$. Put $C^{\prime}=B_{1} \cap\left(v+\varepsilon a+B_{2}\right)$. From the fact that $C^{\prime}$ is homothetic to $B$ and from the relations $\left\|p^{\prime}-q^{\prime}\right\|=\left\|p^{\prime \prime}-q^{\prime \prime}\right\|$,

$$
\left(v+\varepsilon a+B_{2}\right) \cap\left(v+\varepsilon a+H_{p}\right)=\left\{p^{\prime \prime}\right\}, \quad\left(v+\varepsilon a+B_{2}\right) \cap\left(v+\varepsilon a+H_{q}\right)=\left\{q^{\prime \prime}\right\}
$$

we conclude that $C^{\prime}$ is a translate of $C$. At the same time, as is easily seen, the segment $m^{\prime}=(v+\varepsilon a+l) \cap C^{\prime}$ is shorter than $m$, a contradiction.

Lemma 4. Let $B, B_{1}$, and $B_{2}$ satisfy condition (1) of Theorem 2.

(i) If $B$ is a d-polyhedron, then both $B_{1}$ and $B_{2}$ are d-polyhedra and $N\left(B_{1}\right) \cup$ $N\left(B_{2}\right) \subset N(B)$.

(ii) If $B_{1}$ is a d-polytope, then $B$ is a d-polytope with $N(B)=N\left(B_{1}\right)$.

Proof. (i) Let $x$ be a regular point of $B_{1}$ and let $H$ be the hyperplane supporting $B_{1}$ at $x$. Denote by $e$ the outward unit normal to $B_{1}$ at $x$. Choose a translate $v+B_{2}$ that contains $x$ in its interior and consider the body $C=B_{1} \cap\left(v+B_{2}\right)$. Clearly, $x$ is a regular point of $C$ and $e$ is the outward unit normal to $C$ at $x$. Since $C$ is homothetic to $B$, we have $e \in N(C)=N(B)$. As a result, $N\left(B_{1}\right) \subset N(B)$. In particular, $N\left(B_{1}\right)$ is finite and $B_{1}$ is a $d$-polyhedron. Similarly, $N\left(B_{2}\right) \subset N(B)$ and $B_{2}$ is a $d$-polyhedron.

(ii) Let $x$ be a regular point of $B$ and let $H$ be the hyperplane supporting $B$ at $x$. Denote by $H_{1}$ the hyperplane parallel to $H$ and supporting $B_{1}$ such that $B_{1}$ lies on the same side of $H_{1}$ as $B$ does with respect to $H$. Choose points $x_{1} \in B_{1} \cap H_{1}$ and $v \in E^{d}$ with the property $x_{1} \in \operatorname{int}\left(v+B_{2}\right)$ and consider the intersection $C=B_{1} \cap\left(v+B_{2}\right)$. Clearly, $H_{1}$ supports $C$. Since $C$ is homothetic to $B$ and $H_{1}$ is parallel to $H$, there is a regular point of $C$ that belongs to $H_{1}$. Obviously, this is possible only if $z$ is a regular point of $B_{1}$. Hence $z$ lies in the relative interior of a facet of $B_{1}$.

As a result, every hyperplane supporting $B$ at a regular point is parallel to one of the $B_{1}$ facets. This implies that $B$ is the intersection of finitely many closed half-spaces. Since $B$ is bounded (being homothetic to $C$ ), we conclude that $B$ is a $d$-polytope and $N(B) \subset N\left(B_{1}\right)$. From here and (i) above it follows that $N(B)=N\left(B_{1}\right)$. 


\section{Proof of Theorem 2}

While proving Theorem 2, we consider the following four cases.

I. Both $B_{1}$ and $B_{2}$ are bounded.

II. $B_{1}$ is bounded and $B_{2}$ is unbounded (the case when $B_{2}$ is bounded and $B_{1}$ is unbounded is similar).

III. Both $B_{1}$ and $B_{2}$ are unbounded and $B$ is bounded.

IV. $B_{1}, B_{2}$, and $B$ are unbounded.

Case I: Both $B_{1}$ and $B_{2}$ are bounded. Obviously, bounded convex bodies $B_{1}$ and $B_{2}$ satisfying condition (2) are homothetic copies of $B$, which has to be a $d$-simplex. Hence $B_{1}$ and $B_{2}$ satisfy condition (2a). In this case, as proved in [7], conditions (1) and (2a) are equivalent.

Case II: $B_{1}$ is bounded and $B_{2}$ is unbounded.

(2) $\Rightarrow$ (1) Since $B_{1}$ is bounded, the body $B$ from condition (2) is a bounded Choquet simplex, whence it is a $d$-simplex. We can represent $B$ in a suitable coordinate system of $E^{d}$ as

$$
B=\left\{\left(\xi_{1}, \ldots, \xi_{d}\right) \in E^{d}: \xi_{1} \geq 0, \ldots, \xi_{d} \geq 0, \xi_{0}+\xi_{1}+\cdots+\xi_{d} \leq 1\right\} .
$$

Obviously, $B_{1}$ and $B_{2}$ satisfy condition (2b), i.e., $B_{1}$ is homothetic to $B$ and $B_{2}$ is a translate of a tangent cone $T_{x}(B)$, where $x$ is a vertex of $B$. Let $B_{1}=a+B$ and $B_{2}=b+T_{x}(B)$ for some vectors $a=\left(a_{1}, \ldots, a_{d}\right), b=\left(b_{1}, \ldots, b_{d}\right) \in E^{d}$. Without loss of generality, we may assume that $x=(0, \ldots, 0)$. Then

$$
\begin{aligned}
& B_{1}=\left\{\left(\xi_{1}, \ldots, \xi_{d}\right) \in E^{d}: \xi_{1} \geq a_{1}, \ldots, \xi_{d} \geq a_{d}, \xi_{1}+\cdots+\xi_{d} \leq 1+a_{1}+\cdots+a_{d}\right\}, \\
& B_{2}=\left\{\left(\xi_{1}, \ldots, \xi_{d}\right) \in E^{d}: \xi_{1} \geq b_{1}, \ldots, \xi_{d} \geq b_{d}\right\} .
\end{aligned}
$$

For an arbitrary vector $v=\left(v_{1}, \ldots, v_{d}\right) \in E^{d}$, one has

$$
\begin{aligned}
B_{1} \cap\left(v+B_{2}\right)= & \left\{\left(\xi_{1}, \ldots, \xi_{d}\right) \in E^{d}: \xi_{1} \geq \max \left\{a_{1}, b_{1}+v_{1}\right\}, \ldots,\right. \\
& \left.\xi_{d} \geq \max \left\{a_{d}, b_{d}+v_{d}\right\}, \xi_{1}+\cdots+\xi_{d} \leq 1+a_{1}+\cdots+a_{d}\right\} .
\end{aligned}
$$

Clearly, the intersection $B_{1} \cap\left(v+B_{2}\right)$ is nonempty if and only if

$$
1+a_{1}+\cdots+a_{d} \geq \max \left\{a_{1}, b_{1}+v_{1}\right\}+\cdots+\max \left\{a_{d}, b_{d}+v_{d}\right\} .
$$

In this case $B_{1} \cap\left(v+B_{2}\right)=w+\lambda B, \lambda \geq 0$, with

$$
\begin{aligned}
w & =\left(\max \left\{a_{1}, b_{1}+v_{1}\right\}, \ldots, \max \left\{a_{d}, b_{d}+v_{d}\right\}\right), \\
\lambda & =1+a_{1}+\cdots+a_{d}-\left(\max \left\{a_{1}, b_{1}+v_{1}\right\}+\cdots+\max \left\{a_{d}, b_{d}+v_{d}\right\}\right) .
\end{aligned}
$$

(1) $\Rightarrow(2)$ This part of the proof is divided into Lemmas 5-7.

Lemma 5. Under condition (1), if there is a vector $v \in E^{d}$ such that $\operatorname{int}\left(v+B_{2}\right)$ contains two distinct exposed points of $B_{1}$, then $B_{1} \subset\left(v+B_{2}\right)$. 
Proof. Assume for a moment that for a certain vector $v \in E^{d}$, the set $\operatorname{int}\left(v+B_{2}\right)$ contains two distinct points $p, q \in \exp B_{1}$ and $v+B_{2}$ does not contain $B_{1}$. Choose a point $s \in B_{1} \backslash\left(v+B_{2}\right)$. Let $H_{p}$ and $H_{q}$ be some hyperplanes in $E^{d}$ satisfying the conditions $B_{1} \cap H_{p}=\{p\}$ and $B_{1} \cap H_{q}=\{q\}$. One can find a number $\varepsilon>0$ so small that the new translate $v+\varepsilon(p-s)+B_{2}$ still contains both points $p$ and $q$ in its interior.

Now consider the bodies $C=B_{1} \cap\left(v+B_{2}\right)$ and $C^{\prime}=B_{1} \cap\left(v+\varepsilon(p-s)+B_{2}\right)$. By condition (1), both $C$ and $C^{\prime}$ are homothetic to $B$. From here and the relations

$$
C \cap H_{p}=C^{\prime} \cap H_{p}=\{p\}, \quad C \cap H_{q}=C^{\prime} \cap H_{q}=\{q\}
$$

it follows that $C=C^{\prime}$. The last is in contradiction with the fact that the segment $[s, p] \cap C$ is longer than the segment $[s, p] \cap C^{\prime}$.

Lemma 6. Under condition (1), both $B$ and $B_{1}$ are a d-polytopes and $B_{2}$ is a $d$ polyhedron.

Proof. Assume, for contradiction, that $B_{1}$ is not a $d$-polytope. Then the set $\exp B_{1}$ is infinite and has an accumulation point, $a$. Choose a vector $v \in E^{d}$ such that $a \in$ $\operatorname{int}\left(v+B_{2}\right)$. One can find a pair $x, z$ of exposed points of $B_{1}$ so close to $a$ that both $x$ and $z$ belong to $\operatorname{int}\left(v+B_{2}\right)$. By Lemma $5, B_{1} \subset v+B_{2}$. From here and condition (1) we conclude that $B_{1}=B_{1} \cap\left(v+B_{2}\right)$ is homothetic to $B$.

Now let $b$ be an exposed point of $B_{2}$ and let $b_{1}, b_{2}, \ldots$ be a sequence of interior points of $B_{2}$ tending to $b$. Clearly, every translate $\left(a-b_{k}\right)+B_{2}, k=1,2, \ldots$, contains $a$ as an interior point, and, as shown above, $B_{1}$ lies in every convex body $\left(a-b_{k}\right)+B_{2}$. By a continuity argument, $(a-b)+B_{2}$ also contains $B_{1}$. Since $a$ is an exposed point of $(a-b)+B_{2}$, there is a hyperplane $H$ such that $H \cap\left(a-b+B_{2}\right)=\{a\}$. Hence $H \cap B_{1}=\{a\}$ and $a$ is an exposed point of $B_{1}$.

Next chose any point $p \in$ int $B_{1}$ and consider the intersection $C=B_{1} \cap\left(p-b+B_{2}\right)$. Clearly, the hyperplane $H^{\prime}=(p-a)+H$ supports $C$ such that $C \cap H^{\prime}=\{p\}$, i.e., $p$ is an exposed point of $C$ correspondent to $a$ in $B_{1}$ (due to the fact that $B_{1}$ is homothetic to $B$ ). Since $p \in$ int $B_{1}$, there is a ball $U_{r}(p)$ that lies in int $B_{1}$, whence the boundary of $C$ in the neighborhood $U_{r}(p)$ is a part of the boundary of the body $(p-b)+B_{2}$. By Lemma $3, p$ is the only exposed point of $(p-b)+B_{2}$ in int $B_{1}$. This implies that $p$ is the only exposed point of $C$ in $U_{r}(p)$. On the other hand, since $C$ is homothetic to $B_{1}$ and since $a$ is an accumulation point of $B_{1}$, the correspondent point $p \in \exp C$ has to be an accumulation point of the set $\exp C$. The obtained contradiction shows that $B_{1}$ is a $d$-polytope.

Being homothetic to $B_{1}$, the body $B$ is also a $d$-polytope. From Lemma 4 it follows that $B_{2}$ is a $d$-polyhedron.

Lemma 7. Under condition (1), $B$ is a d-simplex and $B_{1}, B_{2}$ satisfy condition (2b).

Proof. We will prove this statement by induction on $d=\operatorname{dim} E^{d}$. The case $d=1$ trivially holds. Assume that the statement of Lemma 7 is true for all $d \leq n-1(n \geq 2)$, 
and let $B, B_{1}, B_{2}$ be convex bodies in $E^{n}$ satisfying condition (1) such that $B$ and $B_{1}$ are bounded and $B_{2}$ is unbounded.

By Lemmas 4 and 6, both $B$ and $B_{1}$ are $n$-polytopes with $N(B)=N\left(B_{1}\right)$ and $B_{2}$ is an unbounded $n$-polyhedron with $N\left(B_{2}\right) \subset N(B)$. Let $F_{2}$ be any unbounded facet of $B_{2}$, and denote by $e$ the outward unit normal to $B_{2}$ at $F_{2}$. Denote by $F$ and $F_{1}$ the facets of $B$ and $B_{1}$, respectively, both having the same outward unit normal $e$.

We are going to show that $F_{2}$ is a simplicial $(n-1)$-cone and $F, F_{1}$ are homothetic ( $n-1)$-simplices both satisfying condition (2b) for $d=n-1$. Indeed, denote by $H_{1}$ the hyperplane containing $F_{1}$, and consider the family of translates $v+B_{2}, v \in E^{d}$, all having the facets $v+F_{2}$ in $H_{1}$. Clearly, $F_{1} \cap\left(v+F_{2}\right) \neq \emptyset$ implies $B_{1} \cap\left(v+B_{2}\right) \neq \emptyset$. From condition (1) it follows that the intersections $B_{1} \cap\left(v+B_{2}\right)$ are homothetic copies of $B$, and, as a result, their facets $F_{1} \cap\left(v+F_{2}\right)$ are homothetic copies of $F$. By the inductive assumption, $F$ and $F_{1}$ are homothetic $(n-1)$-simplices and $F_{2}$ is a simplicial $(n-1)$-cone.

From the fact that every unbounded facet of the $n$-polyhedron $B_{2}$ is a simplicial $(n-1)$-cone we easily conclude that $B_{2}$ is a convex $n$-cone.

Our next step is to show that $B$ and $B_{1}$ are homothetic $n$-simplices. In the notation above, let $H_{1}^{\prime}$ be the hyperplane parallel to $H_{1}$ and supporting $B_{1}$ such that $B_{1}$ lies between $H_{1}$ and $H_{1}^{\prime}$. Denote by $F_{1}^{\prime}$ the intersection of $B_{1}$ and $H_{1}^{\prime}$, and let $x$ be any point in $F_{1}^{\prime}$. Choose a point $z \in \operatorname{rint} F_{2}$ and consider the intersection $B^{\prime}=B_{1} \cap\left(x-z+B_{2}\right)$. Clearly, $B^{\prime}$ lies in $H^{\prime}$. Since $B^{\prime}$ is a degenerate homothetic copy of $B$, we have from condition (1) that $B^{\prime}$ is a point. The last is possible only if $F_{1}^{\prime}$ consists of a single point, i.e., $F_{1}^{\prime}=\{x\}$.

By a continuity argument, one can find a number $\varepsilon \in] 0,1$ [ so close to 1 that $x$ is the only vertex of $B_{1}$ contained in the body $\varepsilon(x-z)+B_{2}$. In this case the intersection $C=B_{1} \cap\left(\varepsilon(x-z)+B_{2}\right)$ is a convex bounded $n$-cone with apex $x$ and base $F=$ $B_{1} \cap\left(\varepsilon(x-z)+F_{2}\right)$. Since $C$ is homothetic to $B$, the base $F$ is an $(n-1)$-simplex. Therefore $C$ is an $n$-simplex. As a result, $B$ is an $n$-simplex.

Since $B_{2}$ is a convex $d$-cone and $B_{1}$ is bounded, we can choose a vector $w \in E^{n}$ such that $B_{1} \subset w+B_{2}$. From here one gets $B_{1}=B_{1} \cap\left(w+B_{2}\right)$, i.e., $B_{1}$ is homothetic to $B$. Hence $B_{1}$ is an $n$-simplex.

It remains to show that $B_{2}$ satisfies condition (2b). Indeed, from the fact that $B_{2}$ is a line-free convex $n$-cone, it follows that $N\left(B_{2}\right)$ contains at least $n$ vectors. On the other hand, since $N\left(B_{2}\right)$ is a proper subset of $N(B)$ and $N(B)$ consists of $n+1$ vectors, the set $N\left(B_{2}\right)$ has exactly $n$ vectors. Hence $B_{2}$ is a simplicial $n$-cone of the form $b+T_{e}(B)$, where $e$ is a vertex of $B$.

Case III: $B$ is bounded and both $B_{1}, B_{2}$ are unbounded.

(2) $\Rightarrow$ (1) Obviously, a bounded body $B$ satisfying condition (2) is a $d$-simplex. We can represent $B$ in a suitable coordinate system of $E^{d}$ as

$$
B=\left\{\left(\xi_{1}, \ldots, \xi_{d}\right) \in E^{d}: \xi_{1} \geq 0, \ldots, \xi_{d} \geq 0, \xi_{0}+\xi_{1}+\cdots+\xi_{d} \leq 1\right\}
$$

Since both $B_{1}$ and $B_{2}$ are unbounded, they must satisfy condition (2c), i.e., $B_{1}$ and $B_{2}$ are translates of tangent cones $T_{x}(B)$ and $T_{z}(B)$, where $x$ and $z$ are distinct vertices of $B$. Let $B_{1}=a+T_{x}(B), B_{2}=b+T_{z}(B)$ for some vectors $a=\left(a_{1}, \ldots, a_{d}\right), b=$ $\left(b_{1}, \ldots, b_{d}\right) \in E^{d}$. Without loss of generality, we may assume that $x=(1,0, \ldots, 0)$ 
and $z=(0,0, \ldots, 0)$. Then

$$
\begin{aligned}
& B_{1}=\left\{\left(\xi_{1}, \ldots, \xi_{d}\right) \in E^{d}: \xi_{2} \geq a_{2}, \ldots, \xi_{d} \geq a_{d}, \xi_{1}+\cdots+\xi_{d} \leq 1+a_{1}+\cdots+a_{d}\right\}, \\
& B_{2}=\left\{\left(\xi_{1}, \ldots, \xi_{d}\right) \in E^{d}: \xi_{1} \geq b_{1}, \ldots, \xi_{d} \geq b_{d}\right\} .
\end{aligned}
$$

For an arbitrary vector $v=\left(v_{1}, \ldots, v_{d}\right) \in E^{d}$, one has

$$
\begin{aligned}
B_{1} \cap\left(v+B_{2}\right)=\{ & \left\{\left(\xi_{1}, \ldots, \xi_{d}\right) \in E^{d}: \xi_{1} \geq b_{1}+v_{1}, \xi_{2} \geq \max \left\{a_{2}, b_{2}+v_{2}\right\}, \ldots,\right. \\
& \left.\xi_{d} \geq \max \left\{a_{d}, b_{d}+v_{d}\right\}, \xi_{1}+\cdots+\xi_{d} \leq 1+a_{1}+\cdots+a_{d}\right\} .
\end{aligned}
$$

Clearly, the intersection $B_{1} \cap\left(v+B_{2}\right)$ is nonempty if and only if

$$
1+a_{1}+\cdots+a_{d} \geq b_{1}+v_{1}+\max \left\{a_{2}, b_{2}+v_{2}\right\}+\cdots+\max \left\{a_{d}, b_{d}+v_{d}\right\} .
$$

In this case $B_{1} \cap\left(v+B_{2}\right)=w+\lambda B, \lambda \geq 0$, with

$$
\begin{aligned}
w & =\left(b_{1}+v_{1}, \max \left\{a_{2}, b_{2}+v_{2}\right\}, \ldots, \max \left\{a_{d}, b_{d}+v_{d}\right\}\right), \\
\lambda & =1+a_{1}+\cdots+a_{d}-\left(b_{1}+v_{1}+\max \left\{a_{2}, b_{2}+v_{2}\right\}+\cdots+\max \left\{a_{d}, b_{d}+v_{d}\right\}\right) .
\end{aligned}
$$

$(1) \Rightarrow(2)$ This part of the proof is divided into Lemmas 8-9.

Lemma 8. Under condition (1), both $B_{1}$ and $B_{2}$ are convex $d$-cones.

Proof. Since any nonempty intersection $B_{1} \cap\left(v+B_{2}\right)$ is a bounded set, the characteristic cones $C\left(B_{1}\right)$ and $C\left(B_{2}\right)$ satisfy the equality $C\left(B_{1}\right) \cap C\left(B_{2}\right)=\{0\}$. In this case we can choose a vector $x \in E^{d}$ such that $B_{1}$ and $x+B_{2}$ are disjoint. Without loss of generality, we may assume that $B_{1}$ and $B_{2}$ are disjoint themselves. Then there is a pair of parallel hyperplanes, $H_{1}$ and $H_{2}$, each separating $B_{1}$ and $B_{2}$ such that $H_{1} \cap B_{1}$ and $H_{2} \cap B_{2}$ are exposed points of $B_{1}$ and $B_{2}$, respectively (see [3]). Let $H_{1} \cap B_{1}=\{p\}$ and $H_{2} \cap B_{2}=\{q\}$. From Lemma 3 it follows that there is a real number $r>0$ such that $q$ is the only exposed point of $B_{2}$ in $U_{r}(q)$. From here we conclude that $B_{2}$ is locally conic at $q$, i.e., there is a number $\mu>0$ such that for any point $x \in$ bd $B_{2} \cap U_{\mu}(q)$ the line segment $[q, x]$ lies in bd $B_{2}$. We are going to prove that $B_{2}=T_{q}\left(B_{2}\right)$, i.e., that $B_{2}$ is a $d$-cone with apex $q$.

Assume, for contradiction, that $B_{2}$ is not a $d$-cone with apex $q$. Then we can choose an exposed half-line $l$ of the cone $T_{q}\left(B_{2}\right)$ that does not lie in $B_{2}$. Since $B_{2}$ is locally conic at $q$, the intersection $l \cap B_{2}$ is a nontrivial exposed segment, [q,t], of $B_{2}$. Choose an arbitrary point $v \in$ int $B_{1}$ and consider the body $B_{2}^{\prime}=(v-q)+B_{2}$. By the above, $B_{2}^{\prime}$ is locally conic at $v$ and the segment $[v, w]=(v-q)+[q, t]$ is an exposed segment of $B_{2}^{\prime}$. Let $H$ be a hyperplane in $E^{d}$ with the property $H \cap B_{2}^{\prime}=[v, w]$.

For any real number $\varepsilon \geq 0$, put

$$
\begin{gathered}
B_{2}^{\prime}(\varepsilon)=\varepsilon(v-w)+B_{2}^{\prime}, \quad L(\varepsilon)=\varepsilon(v-w)+[v, w], \\
v(\varepsilon)=\varepsilon(v-w)+v, \quad w(\varepsilon)=\varepsilon(v-w)+w .
\end{gathered}
$$

Clearly, $L(\varepsilon)$ is an exposed line segment of $B_{2}^{\prime}(\varepsilon)$ such that $B_{2}^{\prime}(\varepsilon) \cap H=L(\varepsilon)$. Moreover, $v(\varepsilon)$ and $w(\varepsilon)$ are the endpoints of $L(\varepsilon)$. 
We claim that the segment $L(0)=[v, w]$ does not lie in $B_{1}$. Indeed, assume for a moment that $L(0) \subset B_{1}$. Since $v(0)=v \in$ int $B_{1}$, one can choose a number $\varepsilon>0$ so small that $L(\varepsilon)$ lies in the interior of $B_{1}$. In this case, by an argument similar to that from the proof of Lemma 3, we get a contradiction.

Hence, by a continuity argument, there is a real number $\varepsilon_{1}>0$ such that $w\left(\varepsilon_{1}\right)$ belongs to the boundary of $B_{1}$ and $L\left(\varepsilon_{1}\right)$ intersects int $B_{1}$. As above, $L\left(\varepsilon_{1}\right)$ does not lie in $B_{1}$; whence its endpoint $v\left(\varepsilon_{1}\right)$ is not in $B_{1}$. Continuously decreasing the value of $\varepsilon$, we get a real number $\varepsilon_{0}$ such that $v\left(\varepsilon_{0}\right)$ belongs to bd $B_{1}$ and $L\left(\varepsilon_{0}\right)$ intersects int $B_{1}$. Similarly, continuously increasing the number $\varepsilon \geq \varepsilon_{1}$, we get the value $\varepsilon_{2}$ such that $B_{1} \cap L\left(\varepsilon_{2}\right)=w\left(\varepsilon_{2}\right)$. As a result, the line through $v$ and $w$ intersects the boundary of $B_{1}$ at two points, $v^{*}$ and $w^{*}$, such that $v^{*}=v\left(\varepsilon_{0}\right)=w\left(\varepsilon_{2}\right)$ and $w^{*}=w\left(\varepsilon_{1}\right)$.

Now consider the intersections $C(\varepsilon)=B_{1} \cap B_{2}^{\prime}(\varepsilon), 0 \leq \varepsilon<\varepsilon_{2}$. By condition (1), every $C(\varepsilon)$ is a convex body homothetic to $B$, and $T(\varepsilon)=H \cap C(\varepsilon)$ is an exposed line segment of $C(\varepsilon)$. Clearly, $T(\varepsilon)=L(\varepsilon) \cap C(\varepsilon)$ and

$$
T(\varepsilon)=\left\{\begin{array}{llc}
{\left[v(\varepsilon), w^{*}\right]} & \text { if } \quad 0 \leq \varepsilon \leq \varepsilon_{0} \\
{\left[v^{*}, w^{*}\right]} & \text { if } \quad \varepsilon_{0} \leq \varepsilon \leq \varepsilon_{1} \\
{\left[v^{*}, w(\varepsilon)\right]} & \text { if } \quad \varepsilon_{1} \leq \varepsilon<\varepsilon_{2}
\end{array}\right.
$$

Choose a half-line $m$ with the endpoint 0 , which lies in the characteristic cone $C\left(B_{2}\right)$ and put

$$
M(\varepsilon)= \begin{cases}B_{1} \cap(v(\varepsilon)+m) & \text { if } \quad 0 \leq \varepsilon \leq \varepsilon_{0} \\ B_{1} \cap\left(v^{*}+m\right) & \text { if } \quad \varepsilon_{0} \leq \varepsilon<\varepsilon_{2}\end{cases}
$$

Since $C\left(B_{1}\right) \cap C\left(B_{2}\right)=\{0\}$, each $M(\varepsilon)$ is a line segment. It is easily seen that $M(\varepsilon)$ is a chord of $C(\varepsilon)$ in the direction $m$ and its position is uniquely determined by $T(\varepsilon)$. Moreover, $M(\varepsilon)$ and $T(\varepsilon)$ have a common endpoint: it is $v(\varepsilon)$ if $0 \leq \varepsilon \leq \varepsilon_{0}$, and it is $v^{*}$ if $\varepsilon_{0} \leq \varepsilon<\varepsilon_{2}$. Since $v(0)=v \in$ int $B_{1}$, the length of $M(0)$ is positive.

Now, consider the size of $C(\varepsilon)$ when $\varepsilon$ increases monotonously from 0 to $\varepsilon_{2}$. Since all the bodies $C(\varepsilon)$ are homothetic to $B$, the size of $C(\varepsilon)$ is proportional to the length of $T(\varepsilon)$. Hence the size of $C(\varepsilon)$ increases when $\varepsilon$ increases from 0 to $\varepsilon_{0}$; it remains constant when $\varepsilon$ increases from $\varepsilon_{0}$ to $\varepsilon_{1}$; and it decreases to 0 when $\varepsilon$ increases from $\varepsilon_{1}$ to $\varepsilon_{2}$.

From the definition of the chord $M(\varepsilon)$ it follows that its length should also be proportional to the length of $T(\varepsilon)$. This is not the case since the length of $M(\varepsilon)$ increases to the length of $M\left(\varepsilon_{0}\right)$ when $\varepsilon$ increases from 0 to $\varepsilon_{0}$ and then remains constant when $\varepsilon$ increases from $\varepsilon_{0}$ to $\varepsilon_{2}$. The obtained contradiction shows that our assumption on $B_{2}$ is not true, whence $B_{2}$ is a cone with apex $q$. In a similar way, $B_{1}$ is a convex $d$-cone with apex $q$.

Lemma 9. Under condition (1), both $B_{1}$ and $B_{2}$ are simplicial d-cones and $B$ is a d-simplex.

Proof. By Lemma 8, both $B_{1}$ and $B_{2}$ are convex $d$-cones. Without loss of generality, we may assume that 0 is their common apex. Since any nonempty intersection $B_{1} \cap\left(v+B_{2}\right)$, $v \in E^{d}$, is bounded, we have $B_{1} \cap B_{2}=\{0\}$. Let $H$ be a hyperplane separating $B_{1}$ and 
$B_{2}$ such that $B_{1} \cap H=B_{2} \cap H=\{0\}$. We divide the proof of Lemma 9 into a sequence of claims below.

Claim 1. There exists a line through 0 that lies in $B_{1} \cup B_{2}$.

Proof of Claim 1. Assume, for contradiction, that no line through 0 lies in $B_{1} \cup B_{2}$. Then $B_{1} \cap\left(-B_{2}\right)=\{0\}$. Let $G$ be a hyperplane separating $B_{1}$ and $-B_{2}$ such that $B_{1} \cap G=\left(-B_{2}\right) \cap G=\{0\}$. Then both $B_{1}$ and $B_{2}$ lie in the same closed half-plane determined by $G$ and $B_{1} \cap G=B_{2} \cap G=\{0\}$.

Choose a point $v \in$ int $B_{1}$ and consider the intersection $C_{1}=B_{1} \cap\left(v+B_{2}\right)$. Clearly, the tangent cone $T_{v}\left(C_{1}\right)$ equals $v+B_{2}$. Similarly, for a given point $w \in \operatorname{int} B_{2}$ and the intersection $C_{2}=B_{1} \cap\left(B_{2}-w\right)$, the tangent cone $T_{w}\left(C_{2}\right)$ equals $B_{1}$. Due to condition (1), the bodies $C_{1}$ and $C_{2}$ are homothetic. Then from the definition of $C_{1}$ and $C_{2}$ it follows that $v \in \exp C_{1}$ and $0 \in \exp C_{2}$ correspond to each other. As a result, $T_{v}\left(C_{1}\right)$ is a translate of $T_{w}\left(C_{2}\right)$. The last is impossible since $B_{1}$ is not a translate of $B_{2}$.

Claim 2. No line through 0 that lies in $B_{1} \cup B_{2}$ meets int $B_{1} \cup$ int $B_{2}$.

Proof of Claim 2. Assume, for contradiction, the existence of a line $l$ through 0 that lies in $B_{1} \cup B_{2}$ and meets int $B_{1} \cup$ int $B_{2}$. Let, for example, $l \cap$ int $B_{1} \neq \emptyset$. Then $l$ intersects int $B_{1} \cap\left(-B_{2}\right)$, and, by a continuity argument, there is a line $l^{\prime}$ through 0 that intersects int $B_{1} \cap\left(-\right.$ int $\left.B_{2}\right)$.

Now choose unit vectors $v \in l \cap$ int $B_{1}, v^{\prime} \in l^{\prime} \cap$ int $B_{1}$ and consider the intersections $C=B_{1} \cap\left(v+B_{2}\right), C^{\prime}=B_{1} \cap\left(v^{\prime}+B_{2}\right)$. Since $0 \in C_{1} \cap C_{2}$, both $C_{1}$ and $C_{2}$ contain more than a single point, and, by condition (1), $C$ and $C^{\prime}$ are convex bodies homothetic to $B$.

Clearly, $C$ lies between the hyperplanes $H$ and $v+H$ such that $C \cap H=\{0\}$ and $C \cap(v+H)=\{v\}$. Similarly, $C^{\prime}$ lies between $H$ and $v^{\prime}+H$ such that $C^{\prime} \cap H=\{0\}$ and $C^{\prime} \cap\left(v^{\prime}+H\right)=\left\{v^{\prime}\right\}$. Since $C$ and $C^{\prime}$ are homothetic, one should have that the line segments $[v, 0]$ and $\left[v^{\prime}, 0\right]$ are parallel. This is not the case by the choice of the lines $l$ and $l^{\prime}$. The obtained contradiction proves Claim 2.

Let $l$ be a line through 0 that lies in $B_{1} \cup B_{2}$. By Claim $2, l \subset$ bd $B_{1} \cup$ bd $B_{2}$. Denote by $S_{i}$ the part of bd $B_{i}$ that is $X$-rayed by $l, i=1,2$. Namely, $S_{i}$ is the set of points $x \in \operatorname{bd} B_{i}$ such that the line $x+l$ intersects int $B_{i}$. In Claim 3 we denote by $n_{z}$ the outward unit normal to $B_{i}$ at a regular point $z \in S_{i}$.

Claim 3. Each set $S_{i}, i=1,2$, lies in a hyperplane through 0 .

Proof of Claim 3. Assume, for contradiction, that $S_{1}$ does not lie in a hyperplane. Then one can choose two regular points $v, w \in S_{1}$ with distinct outward unit normals $n_{v}$ and $n_{w}$, respectively. Let $r>0$ be a real number such that the balls $U_{r}\left(n_{v}\right)$ and $U_{r}\left(n_{w}\right)$ are disjoint. Furthermore, let $\mu>0$ be a real number such that $n_{x} \in U_{r}\left(n_{v}\right)$ for any point $x \in S \cap U_{\mu}(v)$ and $n_{x} \in U_{r}\left(n_{w}\right)$ for any point $x \in S \cap U_{\mu}(w)$. 
Since $B_{1} \cap\left(v+B_{2}\right)=\{v\}$ and $B_{1} \cap\left(w+B_{2}\right)=\{w\}$, we can choose two points $v^{\prime}, w^{\prime} \in$ int $B_{1}$ so close to $v, w$, respectively, that $B_{1} \cap\left(v^{\prime}+B_{2}\right) \subset U_{\mu}(v)$ and $B_{1} \cap$ $\left(w^{\prime}+B_{2}\right) \subset U_{\mu}(w)$. Put $C_{1}=B_{1} \cap\left(v^{\prime}+B_{2}\right)$ and $C_{2}=B_{1} \cap\left(w^{\prime}+B_{2}\right)$. From the consideration above it follows that $v$ is a regular point of $C_{1}$ and $n_{x} \in U_{r}\left(n_{v}\right)$ for any regular point $x$ of $B_{1}$ that lies in the boundary of $C_{1}$. Moreover, any regular point $x$ of $C_{1}$ whose outward unit normal $n_{x}$ to $C_{1}$ satisfies the condition $n_{x} \in U_{r}\left(n_{v}\right)$ is a regular point of $B_{1}$.

By the same argument, $w$ is a regular point of $C_{2}$ and $n_{x} \in U_{r}\left(n_{w}\right)$ for any regular point $x$ of $B_{1}$ that lies in the boundary of $C_{2}$. Also, any regular point $x$ of $C_{2}$ whose outward unit normal $n_{x}$ to $C_{2}$ satisfies the condition $n_{x} \in U_{r}\left(n_{w}\right)$ is a regular point of $B_{1}$.

As a result $U_{r}(v) \cap U_{r}(w)=\emptyset$. This is in contradiction with the fact that both $C_{1}$ and $C_{2}$ are homothetic to $B$. Hence $S_{1}$ lies in a hyperplane through 0 . Similarly, $S_{2}$ lies in a hyperplane through 0 .

Claim 4. The closure $T_{i}=\mathrm{cl} S_{i}$ of $S_{i}$ is a convex $(d-1)$-cone with apex 0 . Moreover, there is a hyperplane $D$ supporting both $B_{1}$ and $B_{2}$ such that $B_{1} \cup B_{2}$ lies in a closed half-space determined by $D$ and the intersection $D \cap$ bd $B_{i}$ is an exposed half-line of $B_{i}, i=1,2$.

Proof of Claim 4. From the definition of $S_{i}$ it easily follows that $0 \in T_{i}$. Since $B_{i}$ is a cone with apex 0 , the set $T_{i}$ is also a cone with apex 0 . From Claim 3 we conclude that $T_{i}$ is a convex $(d-1)$-cone.

Denote by $M$ the hyperplane through 0 orthogonal to the line $l$. If the orthogonal projection $R_{i}$ of $B_{i}$ onto $M$ contained a line, then $T_{i}$ would also contain a line. The last is impossible because $B_{i}$ is line-free. Hence $R_{i}$ is a line-free convex cone and its closure cl $R_{i}$ is a convex acute cone in $M$.

By the above, there is a hyperplane $D$ containing $l$ such that $\mathrm{cl} R_{1} \cap D=\{0\}$ and, as a result, $B_{1} \cap D=\left\{l_{1}\right\}$. In other words, $l_{1}$ is an exposed half-line of $B_{1}$. It remains to show that $D \cap B_{2}$ is an exposed half-line of $B_{2}$. For this, choose any points $v \in$ int $B_{1}$, $w \in$ int $B_{2}$ and consider the intersections $C^{\prime}=B_{1} \cap\left(v+B_{2}\right), C^{\prime \prime}=B_{1} \cap\left(-w+B_{2}\right)$. Clearly, the hyperplane $D-w$ supports $C^{\prime \prime}$ such that $(D-w) \cap C^{\prime \prime}$ is an exposed line segment of $C^{\prime \prime}$ parallel to $l$. Since $C^{\prime}$ and $C^{\prime \prime}$ are homothetic bodies, $v+D$ supports $C^{\prime}$ along an exposed line segment parallel to $l$. Hence $D \cap B_{2}$ is an exposed half-line of $B_{2}$.

Claim 5. $B_{i}=\operatorname{conv}\left(l_{i} \cup T_{i}\right), i=1,2$.

Proof of Claim 5. Choose any points $v \in$ int $B_{1}$ and $w \in$ int $B_{2}$ so close to the facets $T_{1}$ and $T_{2}$, respectively, that $v+B_{2}$ intersects the boundary of $B_{1}$ within $\operatorname{rint} T_{1}$ and $B_{1}$ intersects the boundary of $B_{2}-w$ within rint $T_{2}-w$. Now consider the intersections $C^{\prime}=B_{1} \cap\left(v+B_{2}\right)$ and $C^{\prime \prime}=B_{1} \cap\left(B_{2}-w\right)$. From the choice of $v$ and $w$ it follows that $C^{\prime}$ is a bounded convex cone with apex $w$ and base $T_{1} \cap\left(v+B_{2}\right)$. Similarly, $C^{\prime \prime}$ is a bounded convex cone with apex $w$ and base $\left(T_{2}-w\right) \cap B_{1}$. Due to condition (1), $C^{\prime}$ and $C^{\prime \prime}$ are homothetic. From here we conclude that any exposed half-line of $B_{1}$ is in 
$l_{1} \cup T_{1}$. Similarly, any exposed half-line of $B_{2}$ is in $l_{2} \cup T_{2}$. Hence $B_{i}=\operatorname{conv}\left(l_{i} \cup T_{i}\right)$, $i=1,2$.

Claim 6. Both $B_{1}$ and $B_{2}$ are simplicial d-cones.

Proof of Claim 6. Denote by $L$ the hyperplane containing the facet $T_{1}$ of $B_{1}$, and let $B_{2}^{\prime}$ be any $(d-1)$-dimensional intersection of the form $T_{1} \cap\left(v+B_{2}\right), v \in E^{d}$. Since $0 \in L$, we have $w+B_{2}^{\prime}=L \cap\left(v+w+B_{2}\right)$ for any vector $w \in L$.

If $T_{1}$ and $w+B_{2}^{\prime}$ have nonempty intersection, then $B_{1}$ and $v+w+B_{2}$ have nonempty intersection. Since $B_{1} \cap\left(v+w+B_{2}\right)$ is homothetic to $B$, we conclude that all nonempty intersections of the form $T_{1} \cap\left(w+B_{2}^{\prime}\right), w \in L$, are homothetic to $B_{2}^{\prime}$. According to Case II proved above, $B_{2}^{\prime}$ is a $(d-1)$-simplex and $T_{1}$ is a simplicial $(d-1)$-cone. From here and Claim 5 we conclude that $B_{1}$ is a simplicial $d$-cone. Similarly, $B_{2}$ is a simplicial $d$-cone.

Claim 7. B is a d-simplex.

Proof of Claim 7. Choosing a point $v \in$ int $B_{1}$ so close to $T_{1}$ that $v+B_{2}$ intersects the boundary of $B_{1}$ within $T_{1}$ we get that the intersection $B_{1} \cap\left(v+B_{2}\right)$ is a bounded convex cone with apex $v$ and base $W=T_{1} \cap\left(v+B_{2}\right)$. As proved in Claim $6, W$ is a $(d-1)$-simplex. Hence the intersection $B_{1} \cap\left(v+B_{2}\right)$ is a $d$-simplex. Finally, $B$ is a $d$-simplex.

Case IV: The bodies $B, B_{1}$, and $B_{2}$ are unbounded.

(2) $\Rightarrow$ (1) If unbounded bodies $B, B_{1}, B_{2}$ satisfy condition (2), then $B$ is a simplicial $d$-cone and both $B_{1}, B_{2}$ are translates of $B$ (this derives from either of the conditions (2a) or (2b)). We can represent $B$ in a suitable coordinate system of $E^{d}$ as

$$
B=\left\{\left(\xi_{1}, \ldots, \xi_{d}\right) \in E^{d}: \xi_{1} \geq 0, \ldots, \xi_{d} \geq 0\right\} .
$$

If $B_{1}=a+B$ and $B_{2}=b+B$, with $a=\left(a_{1}, \ldots, a_{d}\right), b=\left(b_{1}, \ldots, b_{d}\right) \in E^{d}$, then for any vector $v=\left(v_{1}, \ldots, v_{d}\right)$,

$$
B_{1} \cap\left(v+B_{2}\right)=\left\{\left(\xi_{1}, \ldots, \xi_{d}\right) \in E^{d}: \xi_{1} \geq \max \left\{a_{1}, b_{1}+v_{1}\right\}, \ldots, \xi_{d} \geq \max \left\{a_{d}, b_{d}+v_{d}\right\}\right\} .
$$

Equivalently, $B_{1} \cap B_{2}=w+B$, where $w=\left(\max \left\{a_{1}, b_{1}+v_{1}\right\}, \ldots, \max \left\{a_{d}, b_{d}+v_{d}\right\}\right)$.

(1) $\Rightarrow$ (2) Since $B$ is unbounded, its characteristic cone contains a half-line, $l$. Obviously, the characteristic cones of both $B_{1}$ and $B_{2}$ contain $l$; so does the characteristic cone of any nonempty intersection $B_{1} \cap\left(v+B_{2}\right), v \in E^{d}$. From here and condition (1) we conclude that any nonempty intersection $B_{1} \cap\left(v+B_{2}\right), v \in E^{d}$, is $d$-dimensional. The remaining part of the proof is divided into Lemmas 10 and 11.

Lemma 10. Under condition (1), both $B_{1}$ and $B_{2}$ are translates of the same convex d-cone.

Proof. Assume, for contradiction, that the conclusion of Lemma 10 is false. Then one can choose exposed points $v \in \exp B_{1}$ and $w \in \exp B_{2}$ that do not correspond 
to each other. From Lemma 2 it follows that $B$ has distinct exposed points, $x$ and $z$, corresponding to the points $v$ and $w$, respectively. By Lemma 1 , none of the tangent cones $T_{x}(B),(x-z)+T_{z}(B)$ lies in the other. Then none of the tangent cones $T_{v}\left(B_{1}\right)$, $(v-w)+T_{w}\left(B_{2}\right)$ lies in the other.

Consider the intersection $C=B_{1} \cap\left(v-w+B_{2}\right)$. Since $C$ is unbounded by the assumption on $B$, it is a nondegenerate homothetic copy of $B$. Obviously, $v$ is an exposed point of $C$ and $T_{v}(C)=T_{v}\left(B_{1}\right) \cap\left(v-w+T_{w}\left(B_{2}\right)\right)$. By the above, the tangent cone $T_{v}(C)$ is a proper part of each of the cones $T_{v}\left(B_{1}\right),(v-w)+T_{w}\left(B_{2}\right)$. From here and the fact that $B$ and $C$ are homothetic we conclude that $B$ contains an exposed point, $y$, distinct from $x, z$ and corresponding to the exposed point $v \in \exp C$. Moreover, $T_{v}(B) \subset(v-x)+T_{x}(B)$, a contradiction with Lemma 1.

Lemma 11. Under condition (1), $B$ is a simplicial $d$-cone and both $B_{1}$ and $B_{2}$ are translates of $B$.

Proof. By Lemma 10, both $B_{1}$ and $B_{2}$ are translates of the same line-free convex cone. Let $z_{1}$ and $z_{2}$ be the apexes of $B_{1}$ and $B_{2}$, respectively. Then the body $C=$ $B_{1} \cap\left(z_{1}-z_{2}+B_{2}\right)$ equals $B_{1}$, and, by condition (1), it is homothetic to $B$. Hence $B_{1}$ is a translate of $B$. Similarly, $B_{2}$ is a translate of $B$. Summing up, we get that $B$ satisfies equation (1), i.e., $B$ is a Choquet simplex. From [1] it follows that $B$ is a simplicial $d$-cone.

\section{References}

1. J. Bair and R. Fourneau, A characterization of unbounded Choquet simplices, Geom. Dedicata 6 (1977), 95-98.

2. G. Choquet, Unicité des représentations intégrales au moyen de points extrémaux dans les cônes convexes réticulés, C. R. Acad. Sci. Paris 243 (1956), 555-557.

3. M. De Wilde, Some properties of the exposed points of finite-dimensional convex sets, J. Math. Anal. Appl. 99 (1984), 257-264.

4. H. G. Eggleston, B. Grünbaum, and V. Klee, Some semicontinuity theorems for convex polytopes and cell-complexes, Comment. Math. Helv. 39 (1964), 165-188.

5. V. Klee, Extremal structure of convex sets, II, Math. Z. 69 (1958), 90-104.

6. C. A. Rogers and G. C. Shephard, The difference body of a convex body, Arch. Math. (Basel) 8 (1957), 220-233.

7. V. Soltan, A characterization of homothetic simplices, Discrete Comput. Geom. 22 (1999), $193-200$.

8. S. Straszewicz, Über exponierte Punkte abgeschlossener Punktmengen, Fund. Math. 24 (1935), 139-143.

Received June 10, 2002, and in revised form October 11, 2002. Online publication January 30, 2003. 